\title{
Inhibitory Effects of Selected Antituberculosis Drugs on Common Human Hepatic Cytochrome P450 and UDP-glucuronosyltransferase Enzymes ${ }^{\text {[ }}$
}

\author{
Lei Cao, David J. Greenblatt, and Awewura Kwara ${ }^{1}$ \\ Graduate Program in Pharmacology and Experimental Therapeutics, Sackler School of Graduate Biomedical Sciences \\ (L.C., D.J.G.) and Department of Integrative Physiology and Pathobiology (D.J.G.), Tufts University School of Medicine, Boston, \\ Massachusetts; Department of Medicine, Warren Alpert Medical School of Brown University, Providence, Rhode Island (A.K.); \\ and The Miriam Hospital, Providence, Rhode Island (A.K.)
}

Received March 21, 2017; accepted June 22, 2017

\begin{abstract}
The comorbidities of tuberculosis and diseases such as HIV require long-term treatment with multiple medications. Despite substantial in vitro and in vivo information on effects of rifampicin and isoniazid on human CYPs, there is limited published data regarding the inhibitory effects of other anti-TB drugs on human CYPs and UGTs. The inhibitory effects of five first-line anti-TB drugs (isoniazid, rifampicin, pyrazinamide, ethambutol, and rifabutin), and the newly approved bedaquiline, were evaluated for six common human hepatic UGT enzymes (UGT1A1, 1A4, 1A6, 1A9, 2B7 and 2B15) in vitro using HLMs. Pyrazinamide, ethambutol, rifabutin and
\end{abstract}

bedaquiline were also studied for their inhibitory effects on eight of the most common human CYP enzymes (CYP1A2, 2B6, 2C8, 2C9, 2C19, 2D6, 2E1 and 3A). Rifabutin inhibited multiple CYPs to varying degrees in vitro, but with all $\mathrm{IC}_{50}$ values exceeding $25 \mu \mathrm{M}$. Rifabutin and rifampicin also inhibited several human UGTs including UGT1A4. The $K_{i}$ value for rifabutin on human hepatic UGT1A4 was $2 \mu \mathrm{M}$. Finally, the six anti-TB drugs produced minimal inhibition of acetaminophen glucuronidation in vitro. Overall, the findings do not raise major concerns regarding metabolic inhibition of human hepatic CYPs and UGTs by the tested anti-TB drugs.

\section{Introduction}

Tuberculosis is one of the leading causes of morbidity and mortality worldwide. The World Health Organization estimated that in 2015 there were 10.4 million incident TB cases, and 1.4 million deaths from TB, and an additional 0.4 million deaths associated with co-infection with HIV (World Health Organization (WHO), 2016). The comorbidity of TB and other diseases requires treatment with multiple medications. Understanding of potential drug-drug interactions (DDIs) is of importance in planning safe and effective combination therapies.

Isoniazid, rifampicin (or rifampin), pyrazinamide, ethambutol, rifabutin, and rifapentine are the principal first-line anti-TB drugs to treat drugsusceptible tuberculosis (Zumla et al., 2013). Bedaquiline is a novel antimycobacterial agent which was approved by FDA in 2012 to treat multidrug resistant tuberculosis (Worley and Estrada, 2014). Among those, rifampicin is a potent inducer of CYPs and UGTs, as well as the P-glycoprotein transport system both in vitro (Rae et al., 2001; Soars et al., 2004) and clinically (Baciewicz et al., 2013). Rifampicin is reported also to

This work was supported by the Eunice Kennedy Shriver National Institute of Child Health and Human Development at the National Institutes of Health [Grant HD071779].

${ }^{1}$ Current affiliation: University of Florida College of Medicine, Gainesville, Florida.

https://doi.org/10.1124/dmd.117.076034.

S This article has supplemental material available at dmd.aspetjournals.org. be an inhibitor of some human CYPs in vitro (Kajosaari et al., 2005), but its overall effect is enzymatic induction, reducing systemic concentrations of many drugs (Ochs et al., 1981). Compared with rifampicin, rifabutin has less potency as a CYP3A inducer and is used as a substitute for rifampicin in patients receiving protease inhibitor and integrase inhibitor-based antiretroviral therapy (World Health Organization (WHO), 2010; Baciewicz et al., 2013; Zumla et al., 2013). Isoniazid is known as an inhibitor of many human CYPs in vitro (Wen et al., 2002; Polasek et al., 2004) and clinically (Ochs et al., 1981, 1983).

Both the inductive effects of rifampicin and inhibitory effects of isoniazid on human CYPs have been extensively reported in vitro and in vivo. However, the data of their effects on human UGTs is limited. Furthermore, the information on other anti-TB drugs is also limited. In this work, inhibitory effects of isoniazid and rifampicin on human hepatic UGTs were studied; and inhibitory properties of the selected anti-TB drugs, including pyrazinamide, ethambutol, rifabutin, and bedaquiline were also studied in vitro with human hepatic CYP and UGT enzymes. Acetaminophen is widely used as an analgesic and antipyretic agent. Since APAP glucuronidation is the pathway responsible for converting two-thirds of a dose of APAP into non-toxic glucuronide conjugates, we also evaluated the inhibitory effect of the anti-TB drugs on acetaminophen glucuronidation.

\section{Materials and Methods}

Chemicals and solvents were purchased from Sigma-Aldrich Corp (St. Louis, MO) and Fisher Scientific (Pittsburg, PA). Isoniazid [Synonym: 4-Pyridinecarboxylic acid

ABBREVIATIONS: APAP, acetaminophen; CYP, cytochrome P450; DDI, drug-drug interactions; DMSO, dimethyl sulfoxide; FDA, U.S. Food and Drug Administration; HLM, human liver microsome; $\mathrm{K}_{\mathrm{i}}$, the inhibition constant for the inhibitor; $\mathrm{MEOH}$, methanol; $\mathrm{MgCl}_{2}$, magnesium chloride; NADP, nicotinamide adenine dinucleotide phosphate; TB, tuberculosis; UDPGA, uridine $5^{\prime}$-diphosphoglucuronic acid; UGT, UDP-glucuronosyltransferase; WHO, World Health Organization. 
hydrazide], rifampin [Synonym: rifampicin, or 3-(4-Methylpiperazinyliminomethyl)rifamycin SV], pyrazinamide, ethambutol hydrochloride [Synonym: $2,2^{\prime}-(1,2-$ Ethanediyldiimino)bis-1-butanol dihydrochloride], and rifabutin [Synonym: Mycobutin] were purchased from Sigma-Aldrich Corp. Bedaquiline [a mixture of diastereomers, Synonym: 6-Bromo- $\alpha$-[2-(dimethylamino)ethyl]-2-methoxy- $\alpha$-1-naphthalenyl$\beta$-phenyl-3-quinolineethanol] was purchased from Toronto Research Chemicals Inc. (North York, Canada). Water was purified with a Milli-Q system (Millipore Corporation, Milford, MA).

Liver samples from individual human donors with no known liver disease were provided by the International Institute for the Advancement of Medicine (Exton, PA), the Liver Tissue Procurement and Distribution System, University of Minnesota (Minneapolis, MN), or the National Disease Research Interchange (Philadelphia, PA). HLMs were prepared as previously described (von Moltke et al., 1993a; Greenblatt et al., 2011). Fifty-three individual liver microsomal preparations were combined to make a batch of pooled HLMs, by mixing an equal amount of protein from each HLM.

Inhibition Studies on CYP-Mediated Oxidation Using HLMs. Previously published incubation procedures using HLMs (Sonnichsen et al., 1995; Hesse et al., 2000; Giancarlo et al., 2001; von Moltke et al., 2001; Greenblatt et al., 2011) were used with modifications. Briefly, appropriate substrates and positive controls (Table 1) were added to incubation tubes. The anti-TB drugs were individually added in a series of concentrations to separate incubation tubes. Isoniazid, rifampicin, pyrazinamide, and ethambutol were at concentrations of $0,10,60$, $100,200,400,600$ and $1000 \mu \mathrm{M}$; rifabutin was at concentrations of $0,10,60,100$, 200, 400, and $600 \mu \mathrm{M}$, except for CYP2C9 and 2D6 with an extra concentration of $1000 \mu \mathrm{M}$; and bedaquiline was at concentrations of $0,0.78,1.56,3.13,6.25$, $12.5,20$ and $25 \mu \mathrm{M}$. The solvent (methanol) was evaporated to dryness at $40^{\circ} \mathrm{C}$ under mild vacuum conditions. Due to their poor solubility in methanol, propofol (the UGT1A9 substrate) and bedaquiline were prepared in DMSO and added directly to incubation tubes (1\% DMSO v/v). Methanol at $1 \%(\mathrm{v} / \mathrm{v})$ in the final incubation mixture was added to reconstitute the anti-TB compounds (except for bedaquiline) after dryness. The incubation mixtures for CYP-mediated oxidation contained $50 \mathrm{mM}$ phosphate buffer ( $\mathrm{pH} 7.5$ ), $5 \mathrm{mM} \mathrm{MgCl}_{2}, 0.5 \mathrm{mM}$ NADP, isocitrate and an isocitric dehydrogenase regenerating system, and appropriate amounts of the pooled HLMs. The anti-TB drugs were preincubated with HLMs (without the index substrates) for $20 \mathrm{~min}$ at $37^{\circ} \mathrm{C}$, and then followed by another timed incubation with the substrates $(250 \mu \mathrm{l})$. All incubations were performed in duplicate. Initial tests for detecting $\mathrm{IC}_{50}$ shifts were carried out by comparing incubations with $20 \mathrm{~min}$ preincubation to incubations without preincubation. One hundred microliters of acetonitrile (or acidified acetonitrile adjusted with 85\% $\mathrm{H}_{3} \mathrm{PO}_{4}$ for CYP2B6 and 2C9) with internal standards were used to stop the reactions. After centrifugation, the supernatant was transferred to HPLC vials for HPLC-UV or HPLC-fluorescence analysis.

Inhibition Studies on Glucuronidation Using HLMs. Previously described incubation procedures were used with modifications (von Moltke et al., 1993b; Court, 2005, 2010). The incubation mixtures for the glucuronidation studies were prepared with $50 \mathrm{mM}$ phosphate buffer $(\mathrm{pH} 7.5), 5 \mathrm{mM} \mathrm{MgCl}_{2}$, alamethicin
(50 $\mu \mathrm{g} / \mathrm{mg}$ protein), and appropriate amounts of the pooled HLMs. The mixtures were kept on ice for $5 \mathrm{~min}$ before use. UDPGA was freshly prepared separately in the phosphate buffer. The reactions were initiated by addition of the UDPGA solution (a final concentration of $10 \mathrm{mM}$ ) in the incubation mixtures $(100 \mu \mathrm{l})$. All incubations were performed in duplicate. The incubations were conducted without preincubation except for those with $\beta$-estradiol (UGT1A1), trifluoperazine (UGT1A4), and APAP, for which the incubations with 20 min preincubation were also conducted. The reactions were stopped by adding $40 \mu \mathrm{l}$ of acetonitrile (or acidified acetonitrile adjusted with $85 \% \mathrm{H}_{3} \mathrm{PO}_{4}$ for UGT2B7 and APAP glucuronidation) with internal standards to the incubation mixtures. After centrifugation, the supernatant was transferred to HPLC vials for HPLC-UV analysis.

$\mathbf{K}_{\mathbf{i}}$ Value for Reversible Enzymatic Inhibition. Inhibition of UGT1A4 by rifabutin was observed with an $\mathrm{IC}_{50}$ value of $11 \mu \mathrm{M}$, which is low enough to trigger a DDI concern. As there was no $\mathrm{IC}_{50}$ shift with and without preincubation, the experimental design for reversible enzymatic inhibition (Greenblatt et al., 2011) was applied to determine the $K_{i}$ value for rifabutin versus human UGT1A4 using pooled HLMs. Varying concentrations of the index substrate (trifluoperazine) at $0,2,5,10,20,34.2,72.4,144.9$ and $336.6 \mu \mathrm{M}$ were incubated at $37^{\circ} \mathrm{C}$ with pooled HLMs in presence of varying concentrations of the inhibitor (rifabutin), at $0,1.25,5,10,30$, and $60 \mu \mathrm{M}$ respectively. Probenecid at $2.4 \mathrm{mM}$ was used as the positive inhibitory control. After $30 \mathrm{~min}$ incubation, the reactions were stopped with $40 \mu \mathrm{l}$ of acetonitrile (in the incubation mixtures of $100 \mu \mathrm{l}$ ) with the internal standard (phenacetin). After centrifugation, the supernatant was transferred to HPLC vials for HPLC-UV analysis.

\section{Analytical Methods}

Previously described methods, with modifications were used for analysis of the in vitro samples in this study (von Moltke et al., 2001; Court, 2005). The HPLC conditions and detection methods are summarized (Supplemental Table 1). APAP glucuronide generated from the in vitro incubations was analyzed using the previously described method, with modifications (Zhao et al., 2015). Briefly, the HPLC analysis was carried out using a Hydro-RP column ( $4 \mu \mathrm{m}, 250 \times$ $4.6 \mathrm{~mm}$, Synergi Hydro-RP; Phenomenex, Torrance, CA), with a flow rate of $1.2 \mathrm{ml} / \mathrm{min}$. The injection volume was $30 \mu \mathrm{l}$, and the UV detection wavelength was $254 \mathrm{~nm}$. A multistep gradient was started at $96.5 \%$ mobile phase $A$ ( $20 \mathrm{mM}$ potassium phosphate buffer, $\mathrm{pH} 2.2$ ) and $3.5 \%$ mobile phase $B$ (methanol) for $5 \mathrm{~min}$, increased to $16 \% \mathrm{~B}$ during the next $5 \mathrm{~min}$, and reached $20 \% \mathrm{~B}$ at $15 \mathrm{~min}$, then to $40 \% \mathrm{~B}$ at $30 \mathrm{~min}$, followed by a 9 min isocratic run at $100 \%$ mobile phase $C\left(50 \% \mathrm{H}_{2} \mathrm{O}\right.$, $50 \%$ methanol), followed by another $10 \mathrm{~min}$ isocratic run at $3.5 \% \mathrm{~B}$. The integration and quantitation were done with the software Chemistation (Agilent, Santa Clara, CA).

TABLE 1

In vitro systems using HLMs for evaluating inhibitory activities of the selected anti-TB drugs on human CYPs and UGTs

\begin{tabular}{|c|c|c|c|c|c|c|}
\hline Enzyme Isoform & Substrate (Conc.) & Internal standard & Metabolite assayed & Inhibitor & $\begin{array}{l}\text { Protein Conc. } \\
\quad(\mu \mathrm{g} / \mathrm{ml})\end{array}$ & $\begin{array}{l}\text { Incubation } \\
\text { time (min) }\end{array}$ \\
\hline CYP1A2 & Phenacetin $(100 \mu \mathrm{M})$ & 2-acetaminophenol & Acetaminophen & $\alpha$-Naphthoflavone & 250 & 20 \\
\hline CYP3A & Triazolam $(250 \mu \mathrm{M})$ & Phenacetin & $\alpha$-Hydroxytriazolam & Ketoconazole & 250 & 20 \\
\hline CYP2B6 & Bupropion $(80 \mu \mathrm{M})$ & 2-acetaminophenol & Hydroxybupropion & Clopidogrel & 333 & 20 \\
\hline CYP2C8 & Taxol $(25 \mu \mathrm{M})$ & Phenacetin & 6-Hydroxytaxol & Quercetin & 300 & 20 \\
\hline CYP2C9 & Flurbiprofen $(5 \mu \mathrm{M})$ & Naproxen & 4'-Hydroxyflurbiprofen & Sulfaphenazole & 250 & 20 \\
\hline CYP2C19 & S-mephenytoin $(25 \mu \mathrm{M})$ & Phenacetin & 4'-Hydroxymephenytoin & Ticlopidine & 500 & 40 \\
\hline CYP2D6 & Dextromethorphan $(25 \mu \mathrm{M})$ & Pronethalol & Dextrorphan & Quinidine & 250 & 20 \\
\hline CYP2E1 & Chlorzoxazone $(50 \mu \mathrm{M})$ & Phenacetin & 6-Hydroxychlorozoxazone & Diethydithiocarbamate & 250 & 20 \\
\hline UGT1A1 & $\beta$-Estradiol $(100 \mu \mathrm{M})$ & Phenacetin & Estradiol-3-glucuronide & Probenecid & 500 & 30 \\
\hline UGT1A4 & Trifluoperazine $(200 \mu \mathrm{M})$ & Phenacetin & Trifluoperazine-glucuronide & Probenecid & 250 & 30 \\
\hline UGT1A6 & Serotonin $(4 \mathrm{mM})$ & Phenacetin & Serotonin-glucuronide & Probenecid & 50 & 30 \\
\hline UGT1A9 & Propofol $(100 \mu \mathrm{M})$ & Phenacetin & Propofol-glucuronide & Niflumic acid & 250 & 30 \\
\hline UGT2B7 & $3^{\prime}$-azidothymidine (AZT) $(500 \mu \mathrm{M})$ & 3-acetaminophenol & AZT-glucuronide & Probenecid & 500 & 120 \\
\hline UGT2B15 & Oxazepam $(100 \mu \mathrm{M})$ & Phenacetin & S-oxazepam-glucuronide & Niflumic acid & 500 & 120 \\
\hline APAP Glucuronidation & Acetaminophen $(0.6 \mathrm{mM})$ & 3-acetaminophenol & APAP-glucuronide & Probenecid & 500 & 120 \\
\hline
\end{tabular}



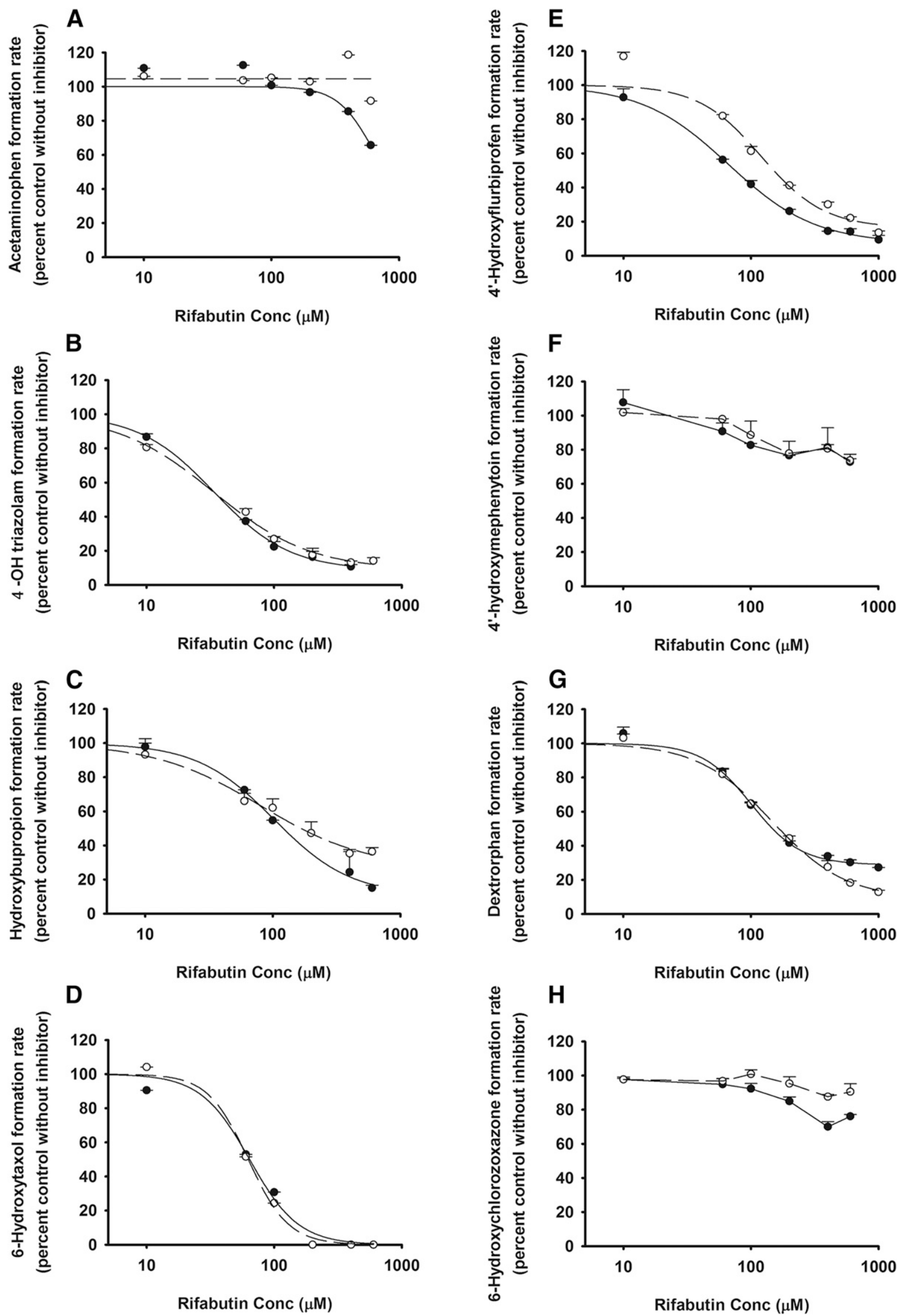

Fig. 1. In vitro inhibitory effects of rifabutin on human hepatic CYPs. (A) CYP1A2, (B) CYP3A, (C) CYP2B6, (D) CYP2C8, (E) CYP2C9, (F) CYP2C19, (G) CYP2D6 and $(\mathrm{H})$ CYP2E1. The incubations were with preincubation (closed circle) and without preincubation (open circle). Data points represent the means \pm S.E. of each concentration of rifabutin that was tested in duplicate. $\mathrm{IC}_{50}$ values were determined by non-linear regression and summarized in Table 2 . 


\section{Data Analysis}

IC $_{50}$ Values. IC $_{50}$ values were determined using nonlinear regression as described previously (von Moltke et al., 2001; Greenblatt et al., 2011). Sigmaplot 11.0 was applied for the nonlinear regression procedure. Briefly, the relationships between the formation of the metabolites of the substrates and the inhibitory concentrations of the tested anti-TB drugs were analyzed by nonlinear regression fitting using eq. 1 . The $\mathrm{IC}_{50}$ values were then generated from the IC values using eq. 2 to take into consideration the possibility of incomplete inhibition.

$$
\begin{gathered}
R=100\left(1-\frac{E_{\max }[I]^{b}}{[I]^{b}+I C^{b}}\right) \\
I C_{50}=\frac{I C}{\left(2 E_{\max }-1\right)^{1 / b}}
\end{gathered}
$$

$R$ is the formation rate of the metabolite of interest, expressed as a percentage fraction of the control reaction velocity with no inhibitor; $E_{\max }$, the maximum degree of inhibition; [I], the concentration of the anti-TB drugs; $b$, an exponent; IC, the inhibitor concentration producing an $R$ value of $50 \%$ of $\left(100-\mathrm{E}_{\max }\right)$, as determined from the nonlinear regression procedure; $\mathrm{IC}_{50}$, the concentration of the tested anti-TB drugs producing $50 \%$ inhibition compared with the inhibitor-free control value, as calculated from the IC value using eq. 2 .

$K_{i}$ Value for Reversible Enzymatic Inhibition. The $K_{i}$ value for rifabutin on human UGT1A4 was determined by nonlinear regression using the reversible inhibition model of full competitive inhibition (Greenblatt et al., 2011) on Sigmaplot 13.0. Significant substrate inhibition of trifluoperazine was observed at the concentration of $336.6 \mu \mathrm{M}$ in our study, as reported previously (Uchaipichat et al., 2006). Thus, the reversible model of full competitive inhibition was fitted using concentrations of trifluoperazine up to $144.9 \mu \mathrm{M}$.

\section{Results}

IC $_{50}$ Values for Rifabutin on Human Hepatic CYPs. Rifabutin inhibited human CYP3A, 2B6, 2D6, 1A2, 2C8 and 2C9 to varying degrees in vitro using the pooled HLMs (Fig. 1; Table 2). At the

highest tested concentration $(600 \mu \mathrm{M})$, no inhibition of human CYP2E1 or 2C19 was observed in vitro with rifabutin (Fig. 1).

IC $_{50}$ Values for Rifabutin on Human Hepatic UGTs, and $K_{i}$ Value for Rifabutin on UGT1A4. Rifabutin inhibited UGT1A1, 1A4 and 2B15 to varying degrees (Figs. 2 and 3; Table 2), and partially inhibited human UGT1A9 and 2B7 (Fig. 2; Table 2) at a high concentration of $600 \mu \mathrm{M}$. The $\mathrm{IC}_{50}$ values for rifabutin on human hepatic UGT1A4 were 10.8 and $11.3 \mu \mathrm{M}$ respectively, for the incubations with and without preincubation. The $\mathrm{K}_{\mathrm{i}}$ value for rifabutin on UGT1A4 using trifluoperazine as the index substrate was $2 \mu \mathrm{M}$, with the pattern of inhibition consistent with reversible competitive inhibition (Fig. 4).

IC $_{50}$ Values for Rifampicin and Isoniazid on Human Hepatic UGTs. Rifampicin had inhibitory effects on UGT1A1, 1A4 and 2B15 with varying $\mathrm{IC}_{50}$ values (Fig. 3); partial inhibition of human UGT1A6 was observed at the highest tested concentration (1000 $\mu \mathrm{M})$ (Fig. 2). No inhibitory effects of isoniazid were observed up to the highest tested concentration (1000 $\mu \mathrm{M})$ (Fig. 2).

Inhibitory Effects of Pyrazinamide, Ethambutol and Bedaquiline on Human Hepatic CYPs and UGTs. Up to the highest tested concentration $(1000 \mu \mathrm{M})$, no significant inhibitory effects of pyrazinamide or ethambutol were observed on the eight screened CYPs (Table 2) or six UGTs (Fig. 2). At the highest tested concentration $(25 \mu \mathrm{M})$, bedaquiline partially inhibited human hepatic CYP3A, 2B6, 2C8, 2C19 and 2D6 at varied levels, but inhibition did not exceed $50 \%$ of the control metabolite formation rate (Fig. 2).

Inhibitory Effects of Anti-TB Drugs on APAP Glucuronidation. The $\mathrm{IC}_{50}$ values for inhibition of APAP glucuronidation by rifabutin, with or without $20 \mathrm{~min}$ preincubation, were 237 and $422 \mu \mathrm{M}$ respectively, and 860 and $545 \mu \mathrm{M}$ respectively for rifampicin. Isoniazid, pyrazinamide, ethambutol, and bedaquiline produced minimal inhibition of APAP glucuronidation (Fig. 5; Table 2). The positive control (probenecid at $0.5 \mathrm{mM}$ ) produced approximately $50 \%$ inhibition of APAP glucuronidation.

\section{Discussion}

Rifabutin inhibited human CYP3A, 2B6, 2C8, 2D6, 1A2, 2C9, UGT1A1, 2B15, UGT1A9 and 2B7 in HLMs, with varying inhibitory potency. However, most of those inhibitory effects observed in vitro are

TABLE 2

\begin{tabular}{|c|c|c|c|c|c|c|c|c|c|c|c|c|}
\hline & \multicolumn{2}{|c|}{ Bedaquiline } & \multicolumn{2}{|c|}{ Rifabutin } & \multicolumn{2}{|c|}{ Pyrazinamide } & \multicolumn{2}{|c|}{ Ethambutol } & \multicolumn{2}{|c|}{ Rifampicin } & \multicolumn{2}{|c|}{ Isoniazid } \\
\hline & $\mathrm{W}^{a} \mu \mathrm{M}$ & $\mathrm{W} / \mathrm{O}^{b} \mu \mathrm{M}$ & $\mathrm{W} \mu \mathrm{M}$ & $\mathrm{W} / \mathrm{O} \mu \mathrm{M}$ & $\mathrm{W} \mu \mathrm{M}$ & $\mathrm{W} / \mathrm{O} \mu \mathrm{M}$ & $\mathrm{W} \mu \mathrm{M}$ & $\mathrm{W} / \mathrm{O} \mu \mathrm{M}$ & $\mathrm{W} \mu \mathrm{M}$ & $\mathrm{W} / \mathrm{O} \mu \mathrm{M}$ & $\mathrm{W} \mu \mathrm{M}$ & $\mathrm{W} / \mathrm{O} \mu \mathrm{M}$ \\
\hline CYP1A2 & $\mathrm{NC}^{c}$ & $\mathrm{NC}$ & $\mathrm{NC}$ & $\mathrm{NC}$ & $\mathrm{NC}$ & $\mathrm{NC}$ & $\mathrm{NC}$ & $\mathrm{NC}$ & - & - & - & - \\
\hline CYP3A & $\mathrm{NC}$ & $\mathrm{NC}$ & 27.9 & 31.5 & $\mathrm{NC}$ & $\mathrm{NC}$ & $\mathrm{NC}$ & $\mathrm{NC}$ & - & - & - & - \\
\hline CYP2B6 & $\mathrm{NC}$ & $\mathrm{NC}$ & 120.8 & 165.3 & $\mathrm{NC}$ & $\mathrm{NC}$ & $\mathrm{NC}$ & $\mathrm{NC}$ & - & - & - & - \\
\hline CYP2C8 & $\mathrm{NC}$ & $\mathrm{NC}$ & 64.0 & 62.6 & $\mathrm{NC}^{d}$ & $\mathrm{NC}$ & $\mathrm{NC}^{e}$ & $\mathrm{NC}$ & - & - & - & - \\
\hline CYP2C9 & $\mathrm{NC}$ & $\mathrm{NC}$ & 75 & 150.9 & $\mathrm{NC}$ & $\mathrm{NC}$ & $\mathrm{NC}$ & $\mathrm{NC}$ & - & - & - & - \\
\hline CYP2C19 & $\mathrm{NC}$ & $\mathrm{NC}$ & $\mathrm{NC}$ & $\mathrm{NC}$ & $\mathrm{NC}$ & $\mathrm{NC}$ & $\mathrm{NC}$ & $\mathrm{NC}$ & - & - & - & - \\
\hline CYP2D6 & $\mathrm{NC}$ & $\mathrm{NC}$ & 147.7 & 166.5 & $\mathrm{NC}$ & $\mathrm{NC}$ & $\mathrm{NC}$ & $\mathrm{NC}$ & - & - & - & - \\
\hline CYP2E1 & $\mathrm{NC}$ & $\mathrm{NC}$ & $\mathrm{NC}$ & $\mathrm{NC}$ & $\mathrm{NC}$ & $\mathrm{NC}$ & $\mathrm{NC}$ & $\mathrm{NC}$ & - & - & - & - \\
\hline UGT1A1 & $\mathrm{NC}$ & $\mathrm{NC}$ & 35 & 44 & $\mathrm{NC}$ & $\mathrm{NC}$ & $\mathrm{NC}$ & $\mathrm{NC}$ & 70 & 63 & $\mathrm{NC}$ & $\mathrm{NC}$ \\
\hline UGT1A4 & $f$ & $\mathrm{NC}$ & 10.8 & 11.3 & - & $\mathrm{NC}$ & - & $\mathrm{NC}$ & - & 230 & - & $\mathrm{NC}$ \\
\hline UGT1A6 & - & $\mathrm{NC}$ & - & $\mathrm{NC}$ & - & $\mathrm{NC}$ & - & $\mathrm{NC}$ & - & $g$ & - & $\mathrm{NC}$ \\
\hline UGT1A9 & - & $\mathrm{NC}$ & - & $\mathrm{NC}$ & - & $\mathrm{NC}$ & - & $\mathrm{NC}$ & - & $\mathrm{NC}$ & - & $\mathrm{NC}$ \\
\hline UGT2B7 & - & $\mathrm{NC}$ & - & $h$ & - & $\mathrm{NC}$ & - & $\mathrm{NC}$ & - & $\mathrm{NC}$ & - & $\mathrm{NC}$ \\
\hline UGT2B15 & - & $\mathrm{NC}$ & - & 81.3 & - & $\mathrm{NC}$ & - & $\mathrm{NC}$ & - & 357 & - & $\mathrm{NC}$ \\
\hline APAP-Glucuronidation & $\mathrm{NC}$ & $\mathrm{NC}$ & 237.2 & 422.2 & $\mathrm{NC}$ & $\mathrm{NC}$ & $\mathrm{NC}$ & $\mathrm{NC}$ & 860 & 545 & $\mathrm{NC}$ & $\mathrm{NC}$ \\
\hline
\end{tabular}

$\mathrm{IC}_{50}$ values of the selected anti-TB drugs on common human hepatic CYPs and UGTs

${ }^{a}$ Incubations with preincubation.

${ }^{b}$ Incubations without preincubation.

${ }^{c}$ Not calculated (No $\mathrm{IC}_{50}$ values were obtained due to less than $50 \%$ inhibition at the highest tested concentrations: $1000 \mu \mathrm{M}$ for rifampicin, pyrazinamide, ethambutol, and isoniazid; $600 \mu \mathrm{M}$ for rifabutin, and $25 \mu \mathrm{M}$ for bedaquiline)

${ }^{d} 41 \%$ inhibition at $1000 \mu \mathrm{M}$.

e $37 \%$ inhibition at $1000 \mu \mathrm{M}$.

not tested.

${ }^{8} 58 \%$ inhibition at $1000 \mu \mathrm{M}$.

${ }^{h} 57 \%$ inhibition at $600 \mu \mathrm{M}$. 


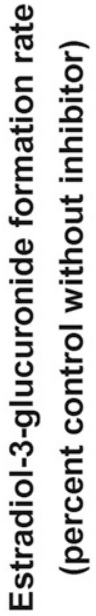

A

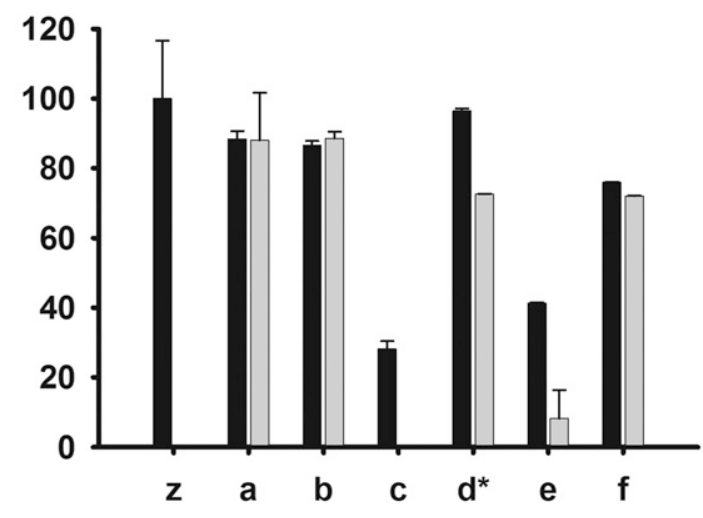

Anti-TB drugs

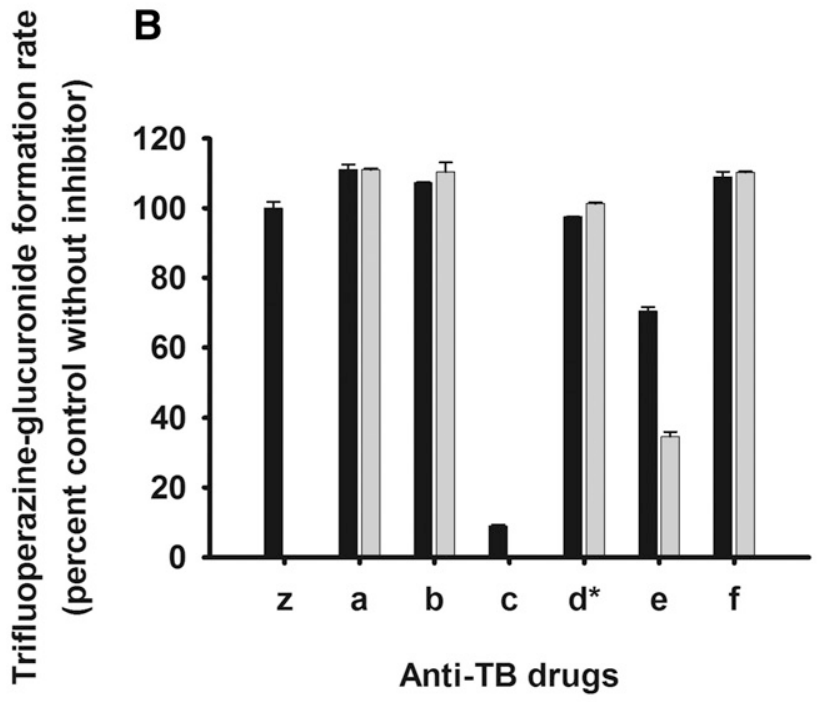

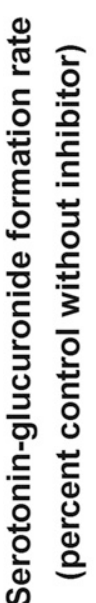

C

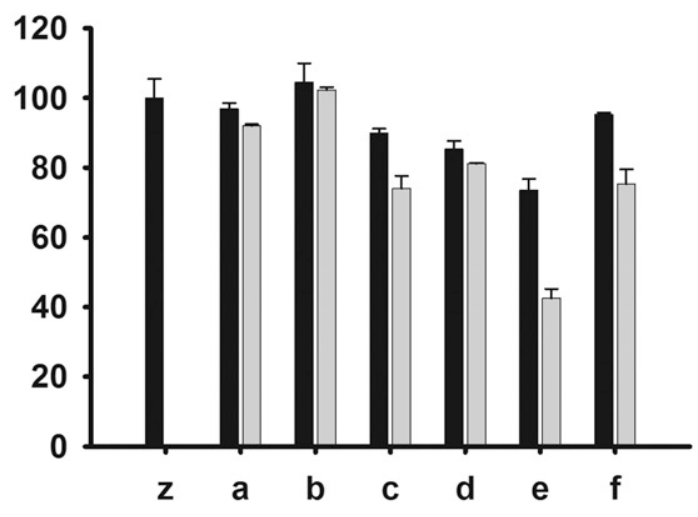

Anti-TB drugs

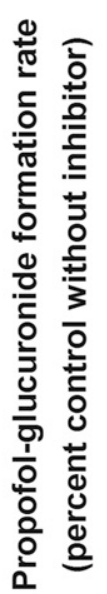

D

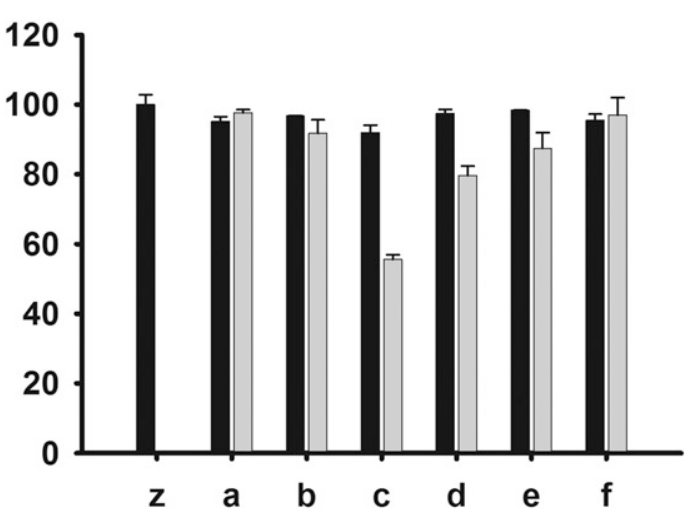

Anti-TB drugs

E
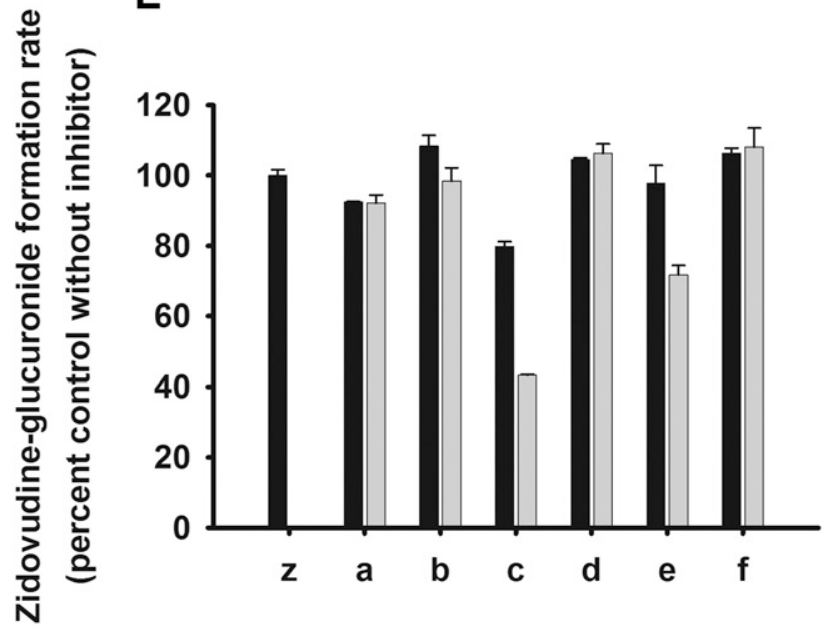

Anti-TB drugs

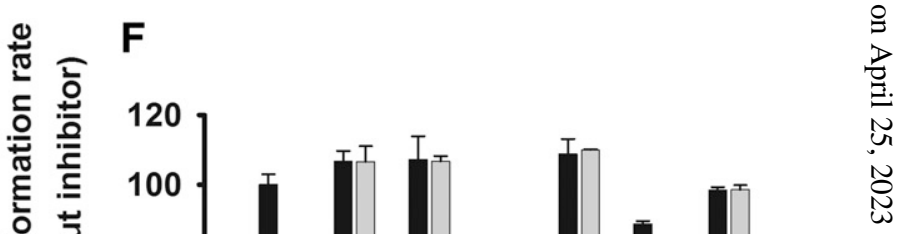

Fig. 2. In vitro inhibitory effects of the selected anti-TB drugs on human hepatic UGTs. (A) UGT1A1, (B) UGT1A4, (C) UGT1A6, (D) UGT1A9, (E) UGT2B7, and (F) UGT2B15. Two concentrations of each compound were tested. z: Control without inhibition (black: $0 \mu \mathrm{M}$ ); a: Pyrazinamide (black:100 $\mu \mathrm{M}$, gray:1000 $\mu \mathrm{M}$ ); b: Ethambutol

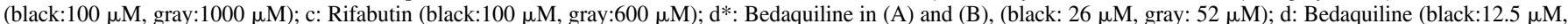
gray: $25 \mu \mathrm{M}$ ); e: Rifampicin (black:100 $\mu \mathrm{M}$, gray:1000 $\mu \mathrm{M}$ ); f: Isoniazid (black:100 $\mu \mathrm{M}$, gray:1000 $\mu \mathrm{M}$ ) 


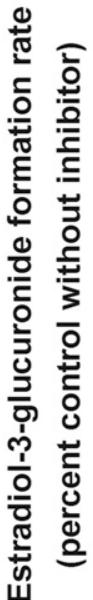

A

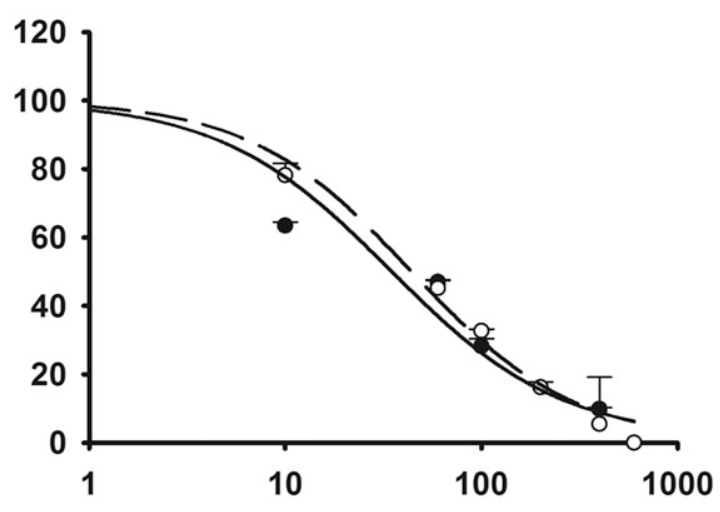

Rifabutin Conc ( $\mu \mathrm{M})$

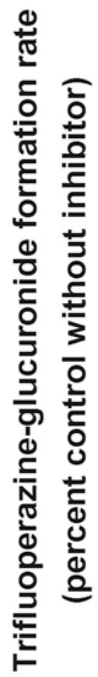

B

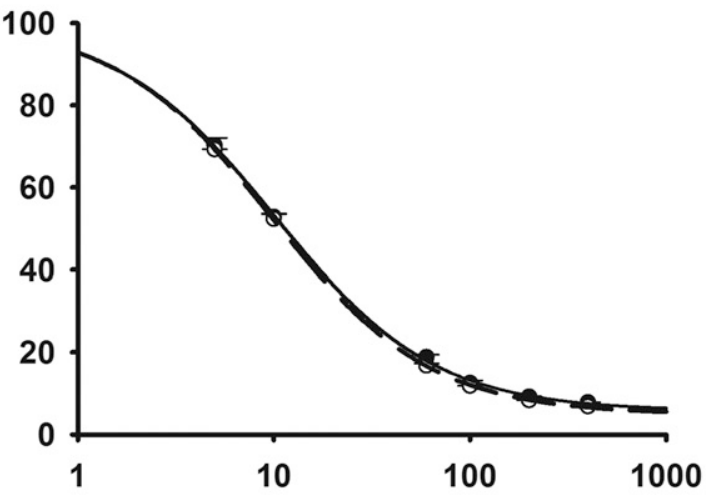

Rifabutin Conc $(\mu \mathrm{M})$

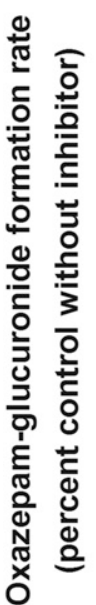

C

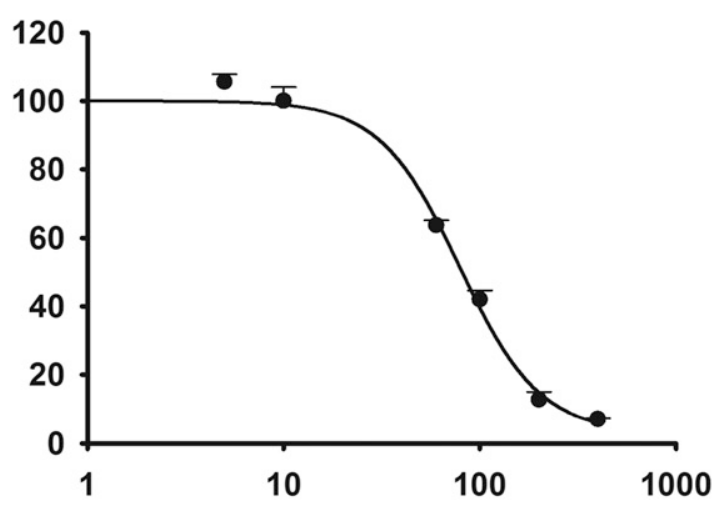

Rifabutin Conc $(\mu \mathrm{M})$

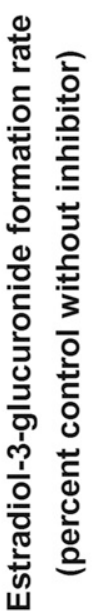

D 120

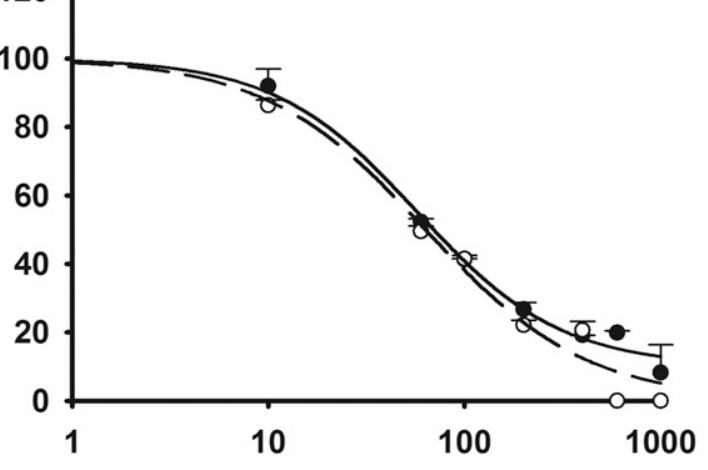

Rifampicin Conc $(\mu \mathrm{M})$

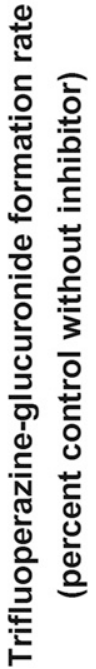

E

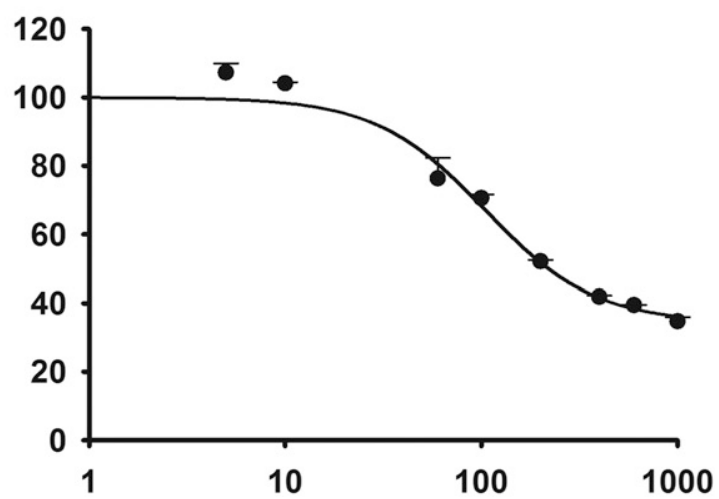

Rifampicin Conc $(\mu \mathrm{M})$

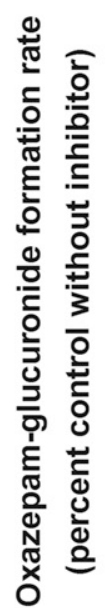

$\mathbf{F}$

120

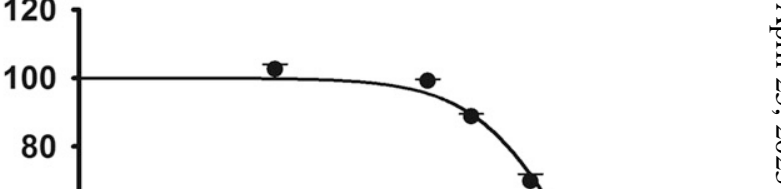

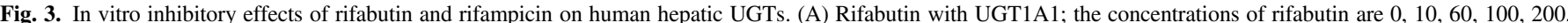

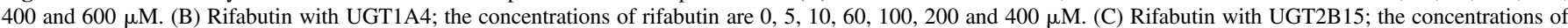

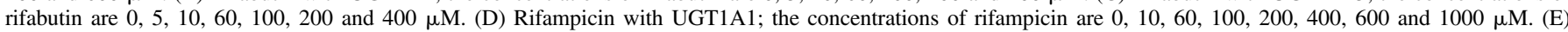

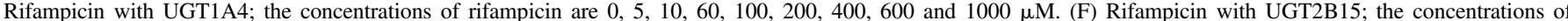

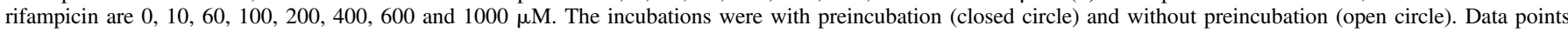
represent the means \pm S.E. of each drug concentration that was tested in duplicate. $\mathrm{IC}_{50}$ values were determined by non-linear regression and summarized in Table 2. 


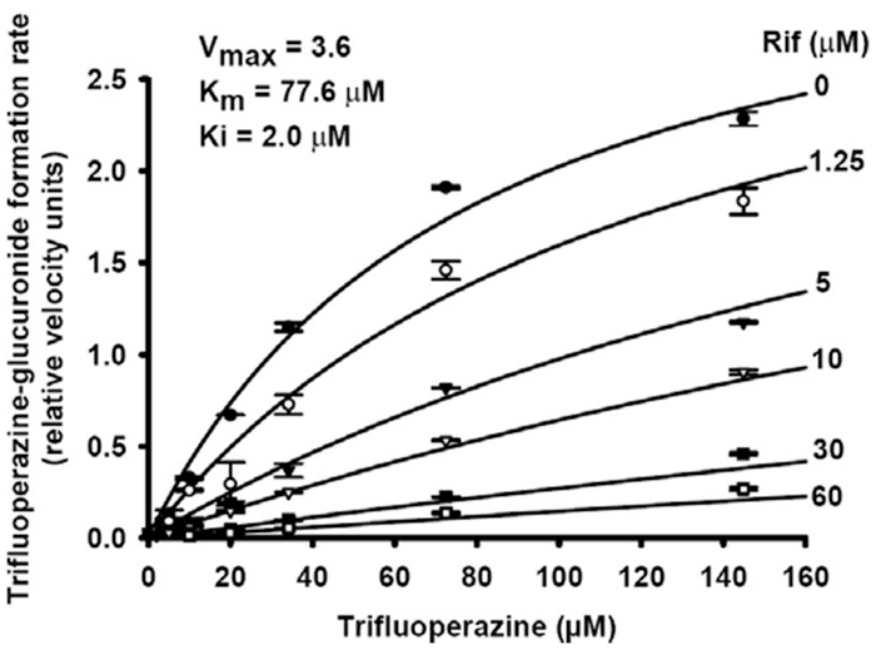

Fig. 4. Rates of formation of trifluoperazine glucuronide in presence of inhibitory rifabutin (Rif). The $\mathrm{K}_{\mathrm{i}}$ value for rifabutin on human hepatic UGT1A4 using trifluoperazine as the index substrate is $2 \mu \mathrm{M}\left(\mathrm{K}_{\mathrm{m}}=77.6 \mu \mathrm{M}, \mathrm{V}_{\max }=3.6\right)$. Data points represent the means \pm S.E. of duplicates.

not likely to be of clinical importance, since the $\mathrm{IC}_{50}$ values were much higher than the reported maximum clinical plasma concentration of rifabutin of approximately $1.1 \mu \mathrm{M}$ (Skinner et al., 1989; Peloquin, 2002).

Rifabutin inhibition of UGT1A4 $\left(\mathrm{K}_{\mathrm{i}}=2 \mu \mathrm{M}\right.$ in pooled HLM) is potentially clinically relevant. Based on FDA guidance (U.S. Department of Health and Human Services Food and Drug Administration Center for Drug Evaluation and Research (CDER), 2012), an approximate estimation of the anticipated clinical DDI was calculated using the ratio of $[\mathrm{I}] / \mathrm{K}_{\mathrm{i}}$ where $[\mathrm{I}]$ is the maximum in vivo plasma concentration of rifabutin $(1.1 \mu \mathrm{M})$ (Skinner et al., 1989) and $\mathrm{K}_{\mathrm{i}}$ was $2 \mu \mathrm{M}$ in this estimation. The ratio of 0.55 indicates a possibility that rifabutin may increase the systemic exposure of some drugs which are metabolized mainly by human UGT1A4. On the other hand, it has been widely reported that rifabutin induces human CYPs and UGTs (Baciewicz et al., 2013). Thus the prediction of the overall drug-drug interaction of rifabutin needs to consider both its inhibitory and possible inductive properties.

Human UGT1A1 is the principal metabolizing enzyme for several anti-HIV drugs such as raltegravir (Kassahun et al., 2007) and dolutegravir (Castellino et al., 2013). The $\mathrm{IC}_{50}$ values for rifabutin and rifampicin on UGT1A1 were approximately 35 and $70 \mu \mathrm{M}$ respectively (Fig. 3). However, the inhibitory effects of rifabutin and rifampicin are not of major clinical importance, as their overall effects show predominantly inductive properties. In clinical studies, rifampicin significantly decreased the systemic exposure of dolutegravir (Dooley et al., 2013). Co-administration of rifabutin, on the other hand, did not alter the pharmacokinetics of raltegravir (Brainard et al., 2011) or dolutegravir (Dooley et al., 2013).

The $\mathrm{IC}_{50}$ values for rifabutin on CYP1A2 demonstrated a leftward shift (smaller $\mathrm{IC}_{50}$ ) between the incubations without and with preincubation, consistent with time-dependent inhibition (Fig. 1). We did not determine the rate constant for inactivation in this study. Nevertheless, to our knowledge, no clinically meaningful DDIs due to inhibition of CYP1A2 by rifabutin have been reported.

The lack of significant inhibition of UGTs by isoniazid is reassuring for the use of isoniazid for latent TB treatment in HIV-infected patient receiving integrase strand transfer inhibitors (INSTIs)-based antiretroviral therapy. Isoniazid for 6 or 9 months is one of the preferred regimens for the treatment of latent TB (Getahun et al., 2015). The INSTIs such as
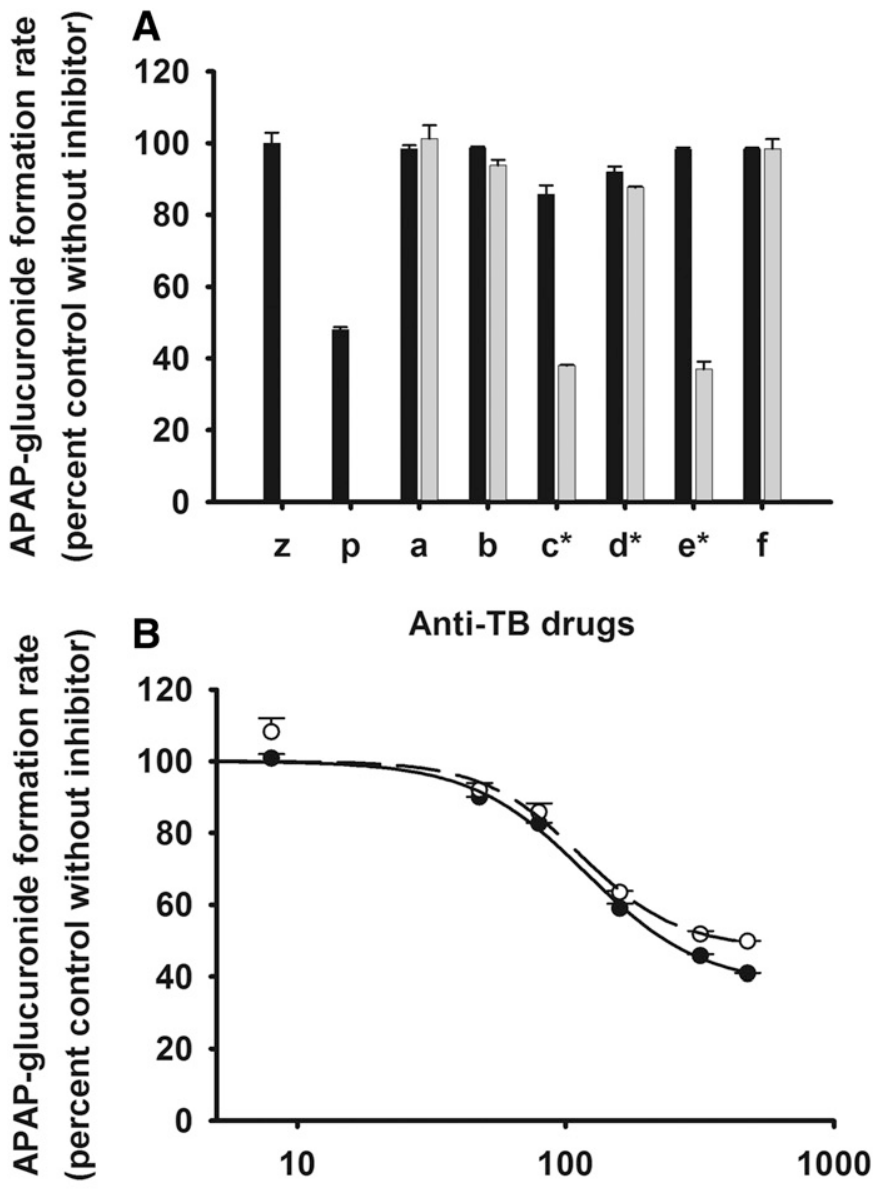

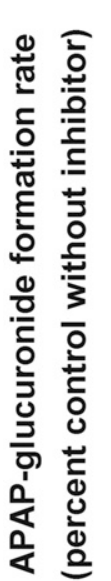

$C$
120
100
80
60
40
20

20
100
80
60
40
20
0

Rifabutin Conc $(\mu \mathrm{M})$

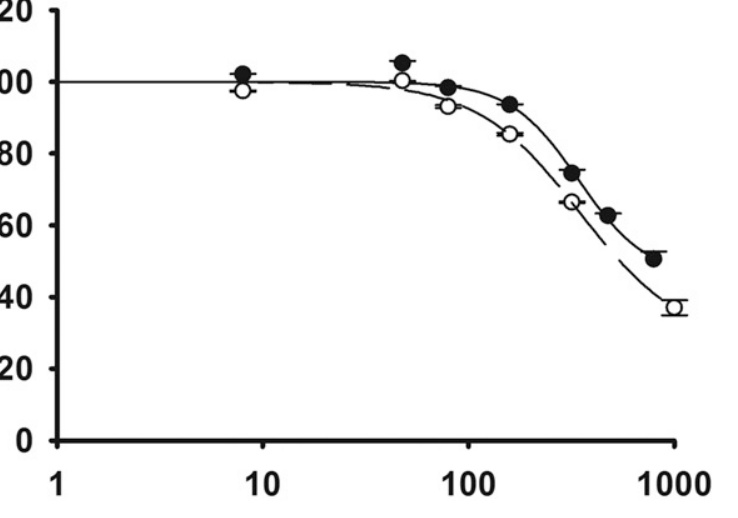

Rifampicin Conc $(\mu \mathrm{M})$

Fig. 5. (A) In vitro inhibitory effects of the tested anti-TB drugs on APAP glucuronidation using HLMs. Two concentrations of each compound were tested. z: Control without inhibitor (black: $0 \mu \mathrm{M}$ ); p: Probenecid as the positive control (0.5 mM); a: Pyrazinamide (black: $100 \mu \mathrm{M}$, gray: $1000 \mu \mathrm{M}$ ); b: Ethambutol (black: $100 \mu \mathrm{M}$, gray:1000 $\mu \mathrm{M}$ ); $\mathrm{c}^{*}$ : Rifabutin (black:80 $\mu \mathrm{M}$, gray:600 $\mu \mathrm{M}$ ); $\mathrm{d}^{*}$ : Bedaquiline (black:26 $\mu \mathrm{M}$, gray:52 $\mu \mathrm{M}$ ); $\mathrm{e}^{*}$ : Rifampicin (black:80 $\mu \mathrm{M}$, gray: $1000 \mu \mathrm{M}$ ); f: Isoniazid (black:100 $\mu \mathrm{M}$, gray:1000 $\mu \mathrm{M}$ ). (B) In vitro inhibitory effects of rifabutin on APAP glucuronidation. The concentrations of rifabutin are 0 , 8, 47.7, 79.6, 159.1, 318.3, and 477.4 $\mu \mathrm{M}$. (C) In vitro inhibitory effects of rifampicin on APAP glucuronidation. The concentrations of rifampicin are 0,8 , 47.7, 79.6, 159.1, 318.3, 477.4 and $795.7 \mu \mathrm{M}$ in incubations with preincubation (closed circle) and $0,8,47.7,79.6,159.1,318.3$, and $1000 \mu \mathrm{M}$ in incubations without preincubation (open circle). Data points represent the means \pm S.E. of each drug concentration that was tested in duplicate. $\mathrm{IC}_{50}$ values were determined by nonlinear regression and summarized in Table 2. 
dolutegravir, raltegravir or elvitegravir that are primarily metabolized by UGTs are essential components of preferred first-line antiretroviral therapy for HIV infection (Günthard et al., 2016). The findings in this in vitro study suggest that no dose adjustment of the INSTIs is necessary when co-administered with isoniazid, although in vivo studies may be needed to confirm this.

Incomplete inhibition was observed for a number of the CYP and UGT isoforms in this study. This might be explained by the participation of multiple isoforms in a given biotransformation pathway, particularly if the substrate is not specific for the target enzyme isoform of interest. The incomplete inhibition in Fig. 5, B and C could be explained by this. However, the explanation for incomplete inhibition in Fig. 3E is not clear, since the substrate trifluoperazineis reported to be highly specific for UGT1A4 (Uchaipichat et al., 2006). In addition, the incomplete inhibition was only observed with rifampicin but not with rifabutin. In any case, the calculated $\mathrm{IC}_{50}$ values correctly represent the inhibitor concentration that reduces the metabolite formation to $50 \%$ of the inhibitor-free control value (fixed at $100 \%$ ). In the scenario of incomplete inhibition ( $E_{\max }$ less than 1.0 in eq. 1$)$, the true $\mathrm{IC}_{50}$ is calculated from IC using eq. 2 .

Since high concentrations of the anti-TB drugs were used in the incubations, $1 \%$ methanol was introduced to improve solubility. Bedaquiline was prepared in DMSO for better solubility and added directly to the incubation mixtures. Because methanol and DMSO may themselves inhibit metabolic activities of CYPs and UGTs, inhibitorfree controls were included using the same concentrations of these solvents to control for any solvent effects that might occur.

Acetaminophen is a common over-the-counter analgesic and antipyretic. The CYP mediated oxidation pathway produces the toxic intermediate $\mathrm{N}$-acetyl-p-benzoquinone imine (Miner and Kissinger, 1979), known to be responsible for acetaminophen hepatotoxicity. Parallel glucuronidation and sulfation of APAP are the major metabolizing pathways for generation of non-toxic metabolite conjugates. Several UGTs, including UGT1A1, 1A6, 1A9 and 2B15 are involved in APAP glucuronidation. (Court and Greenblatt, 2000; Court et al., 2001; Krishnaswamy et al., 2005; Mutlib et al., 2006). None of the selected anti-TB drugs significantly inhibited glucuronidation of APAP in vitro in this study (Fig. 5).

In conclusion, this study provides data on the inhibitory effects of anti-TB drugs on common CYPs and UGTs using HLMs in vitro. Rifabutin and rifampicin showed inhibitory properties to varying degrees. The findings for the other tested anti-TB drugs do not raise new concerns about clinical DDIs involving inhibition of hepatic CYPs and UGTs.

\section{Acknowledgments}

We thank Dr. Michael Court, Washington State University, Pullman, WA, for his advice regarding UDP-glucuronosyltransferase enzymes (UGTs).

\section{Authorship Contributions}

Participated in research design: Greenblatt, Kwara, Cao.

Conducted experiments: Cao.

Contributed new reagents or analytic tools: Greenblatt, Kwara.

Performed data analysis: Cao, Greenblatt, Kwara. Kwara.

Wrote or contributed to the writing of the manuscript: Cao, Greenblatt,

\section{References}

Baciewicz AM, Chrisman CR, Finch CK, and Self TH (2013) Update on rifampin, rifabutin, and rifapentine drug interactions. Curr Med Res Opin 29:1-12.

Brainard DM, Kassahun K, Wenning LA, Petry AS, Liu C, Lunceford J, Hariparsad N, Eisenhandler R, Norcross A, DeNoia EP, et al. (2011) Lack of a clinically meaningful pharmacokinetic effect of rifabutin on raltegravir: in vitro/in vivo correlation. J Clin Pharmacol 51:943-950.
Castellino S, Moss L, Wagner D, Borland J, Song I, Chen S, Lou Y, Min SS, Goljer I, Culp A, et al. (2013) Metabolism, excretion, and mass balance of the HIV-1 integrase inhibitor dolutegravir in humans. Antimicrob Agents Chemother 57:3536-3546.

Court MH (2005) Isoform-selective probe substrates for in vitro studies of human UDPglucuronosyltransferases. Methods Enzymol 400:104-116.

Court MH (2010) Interindividual variability in hepatic drug glucuronidation: studies into the role of age, sex, enzyme inducers, and genetic polymorphism using the human liver bank as a model system. Drug Metab Rev 42:209-224.

Court MH, Duan SX, von Moltke LL, Greenblatt DJ, Patten CJ, Miners JO, and Mackenzie PI (2001) Interindividual variability in acetaminophen glucuronidation by human liver microsomes: identification of relevant acetaminophen UDP-glucuronosyltransferase isoforms. J Pharmacol Exp Ther 299:998-1006.

Court MH and Greenblatt DJ (2000) Molecular genetic basis for deficient acetaminophen glucuronidation by cats: UGT1A6 is a pseudogene, and evidence for reduced diversity of expressed hepatic UGT1A isoforms. Pharmacogenetics 10:355-369.

Dooley KE, Sayre P, Borland J, Purdy E, Chen S, Song I, Peppercorn A, Everts S, Piscitelli S, and Flexner C (2013) Safety, tolerability, and pharmacokinetics of the HIV integrase inhibitor dolutegravir given twice daily with rifampin or once daily with rifabutin: results of a phase 1 study among healthy subjects. J Acquir Immune Defic Syndr 62:21-27.

Getahun H, Chaisson RE, and Raviglione M (2015) Latent Mycobacterium tuberculosis infection. N Engl J Med 373:1179-1180.

Giancarlo GM, Venkatakrishnan K, Granda BW, von Moltke LL, and Greenblatt DJ (2001) Relative contributions of CYP2C9 and 2C19 to phenytoin 4-hydroxylation in vitro: inhibition by sulfaphenazole, omeprazole, and ticlopidine. Eur J Clin Pharmacol 57:31-36.

Greenblatt DJ, Zhao Y, Venkatakrishnan K, Duan SX, Harmatz JS, Parent SJ, Court MH, and von Moltke LL (2011) Mechanism of cytochrome P450-3A inhibition by ketoconazole. J Pharm Pharmacol 63:214-221.

Günthard HF, Saag MS, Benson CA, del Rio C, Eron JJ, Gallant JE, Hoy JF, Mugavero MJ, Sax PE, Thompson MA, et al. (2016) Antiretroviral drugs for treatment and prevention of HIV infection in adults: 2016 recommendations of the international antiviral society-USA panel. JAMA 316:191-210.

Hesse LM, Venkatakrishnan K, Court MH, von Moltke LL, Duan SX, Shader RI, and Greenblatt DJ (2000) CYP2B6 mediates the in vitro hydroxylation of bupropion: potential drug interactions with other antidepressants. Drug Metab Dispos 28:1176-1183.

Kajosaari LI, Laitila J, Neuvonen PJ, and Backman JT (2005) Metabolism of repaglinide by CYP2C8 and CYP3A4 in vitro: effect of fibrates and rifampicin. Basic Clin Pharmacol Toxicol 97:249-256.

Kassahun K, McIntosh I, Cui D, Hreniuk D, Merschman S, Lasseter K, Azrolan N, Iwamoto M, Wagner JA, and Wenning LA (2007) Metabolism and disposition in humans of raltegravir (MK-0518), an anti-AIDS drug targeting the human immunodeficiency virus 1 integrase enzyme. Drug Metab Dispos 35:1657-1663.

Krishnaswamy S, Hao Q, Al-Rohaimi A, Hesse LM, von Moltke LL, Greenblatt DJ, and Court MH (2005) UDP glucuronosyltransferase (UGT) 1 A6 pharmacogenetics: II. Functional impact of the three most common nonsynonymous UGT1A6 polymorphisms (S7A, T181A, and R184S). $J$ Pharmacol Exp Ther 313:1340-1346.

Miner DJ and Kissinger PT (1979) Evidence for the involvement of N-acetyl-p- quinoneimine in acetaminophen metabolism. Biochem Pharmacol 28:3285-3290.

Mutlib AE, Goosen TC, Bauman JN, Williams JA, Kulkarni S, and Kostrubsky S (2006) Kinetics of acetaminophen glucuronidation by UDP-glucuronosyltransferases 1A1, 1A6, 1A9 and 2B15. Potential implications in acetaminophen-induced hepatotoxicity. Chem Res Toxicol 19:701-709.

Ochs HR, Greenblatt DJ, and Knüchel M (1983) Differential effect of isoniazid on triazolam oxidation and oxazepam conjugation. Br J Clin Pharmacol 16:743-746.

Ochs HR, Greenblatt DJ, Roberts GM, and Dengler HJ (1981) Diazepam interaction with antituberculosis drugs. Clin Pharmacol Ther 29:671-678.

Peloquin CA (2002) Therapeutic drug monitoring in the treatment of tuberculosis. Drugs 62 : 2169-2183.

Polasek TM, Elliot DJ, Lewis BC, and Miners JO (2004) Mechanism-based inactivation of human cytochrome P4502C8 by drugs in vitro. J Pharmacol Exp Ther 311:996-1007.

Rae JM, Johnson MD, Lippman ME, and Flockhart DA (2001) Rifampin is a selective, pleiotropic inducer of drug metabolism genes in human hepatocytes: studies with cDNA and oligonucleotide expression arrays. J Pharmacol Exp Ther 299:849-857.

Skinner MH, Hsieh M, Torseth J, Pauloin D, Bhatia G, Harkonen S, Merigan TC, and Blaschke TF (1989) Pharmacokinetics of rifabutin. Antimicrob Agents Chemother 33:1237-1241.

Soars MG, Petullo DM, Eckstein JA, Kasper SC, and Wrighton SA (2004) An assessment of udpglucuronosyltransferase induction using primary human hepatocytes. Drug Metab Dispos 32: 140-148.

Sonnichsen DS, Liu Q, Schuetz EG, Schuetz JD, Pappo A, and Relling MV (1995) Variability in human cytochrome P450 paclitaxel metabolism. J Pharmacol Exp Ther 275:566-575.

U.S. Department of Health and Human Services Food and Drug Administration Center for Drug Evaluation and Research (CDER) (2012) Guidance for Industry Drug Interaction Studies - Study Design, Data Analysis, Implications for Dosing, and Labeling Recommendations, Draft Guidance. CDER, Silver Spring, Maryland.

Uchaipichat V, Mackenzie PI, Elliot DJ, and Miners JO (2006) Selectivity of substrate (trifluoperazine) and inhibitor (amitriptyline, androsterone, canrenoic acid, hecogenin, phenylbutazone, quinidine, quinine, and sulfinpyrazone) "probes" for human udp-glucuronosyltransferases. Drug Metab Dispos 34:449-456.

von Moltke LL, Greenblatt DJ, Giancarlo GM, Granda BW, Harmatz JS, and Shader RI (2001) Escitalopram (S-citalopram) and its metabolites in vitro: cytochromes mediating biotransformation, inhibitory effects, and comparison to R-citalopram. Drug Metab Dispos 29: 1102-1109.

von Moltke LL, Greenblatt DJ, Harmatz JS, and Shader RI (1993a) Alprazolam metabolism in vitro: studies of human, monkey, mouse, and rat liver microsomes. Pharmacology 47: 268-276.

von Moltke LL, Manis M, Harmatz JS, Poorman R, and Greenblatt DJ (1993b) Inhibition of acetaminophen and lorazepam glucuronidation in vitro by probenecid. Biopharm Drug Dispos 14:119-130.

Wen X, Wang JS, Neuvonen PJ, and Backman JT (2002) Isoniazid is a mechanism-based inhibitor of cytochrome P450 1A2, 2A6, 2C19 and 3A4 isoforms in human liver microsomes. Eur J Clin Pharmacol 57:799-804. 
World Health Organization (WHO). (2010) Guidelines for Treatment of Tuberculosis. 4th ed. WHO Press, Geneva, Switzerland.

World Health Organization (WHO). (2016) Global Tuberculosis Report 2016. WHO Press, Geneva, Switzerland.

Worley MV and Estrada SJ (2014) Bedaquiline: a novel antitubercular agent for the treatment of multidrug-resistant tuberculosis. Pharmacotherapy 34:1187-1197.

Zhao Y, Harmatz JS, Epstein CR, Nakagawa Y, Kurosaki C, Nakamura T, Kadota T, Giesing D, Court MH, and Greenblatt DJ (2015) Favipiravir inhibits acetaminophen sulfate formation but minimally affects systemic pharmacokinetics of acetaminophen. Br J Clin Pharmacol 80: $1076-1085$.
Zumla A, Nahid P, and Cole ST (2013) Advances in the development of new tuberculosis drugs and treatment regimens. Nat Rev Drug Discov 12:388-404.

Address correspondence to: Dr. David J. Greenblatt, Tufts University School of Medicine, 136 Harrison Avenue, Boston, MA 02111. E-mail: DJ.Greenblatt@Tufts. edu 